\title{
Análisis de canales digitales para la distribución de material educativo que promueva el consumo saludable de productos agroecológicos en estudiantes de la ESPOCH
}

Analysis of digital channels for the distribution of educational material that promotes the healthy consumption of agroecological products in ESPOCH students

Ruth Genoveva Barba Vera. ${ }^{1}$, Alba Isabel Maldonado Núñez. ${ }^{2}$ \& Edison Fernando Martínez Espinoza. ${ }^{3}$

\begin{abstract}
.
DOI: https://doi.org/10.33262/concienciadigital.v4i3.1.1829

Introduction. Within the framework of the multidisciplinary project of linking "ESPOCH, Latin American University for Fair Trade", the Faculty of Informatics and Electronics of the Escuela Superior Politecnica de Chimborazo (ESPOCH), planned the development of educational material to educate polytechnic students about of fair trade and motivate them to learn about agroecological products using digital channels. Target. Development of AN EDUCATIONAL MATERIAL ABOUT THE HEALTHY CONSUMPTION OF PRODUCTS FROM THE MAQUITA FOUNDATION. Methodology. A three-phase educational material design process was proposed, starting in phase 1 of the selection of an adequate educational methodology for which a Systematic Literature Review (RSL) was applied, in phase 2 the educational material to

\footnotetext{
${ }^{1}$ Escuela Superior Politécnica de Chimborazo, Ingeniería en Control y Redes Industriales, Chimborazo, rbarba@espoch.edu.ec, https://orcid.org/0000-0003-0272-171X

${ }^{2}$ Escuela Superior Politécnica de Chimborazo, Ingeniería en Telecomunicaciones, Riobamba, Chimborazo, alba.maldonado@espoch.edu.ec, https://orcid.org/0000-0001-8673-0319

${ }^{3}$ Escuela Superior Politécnica de Chimborazo, Licenciatura en Diseño Gráfico, Chimborazo, Ecuador. edmartinez@espoch.edu.ec, https://orcid.org/0000-0002-5320-9460
} 
be developed was determined and in phase 3 the plan for the dissemination of educational material was proposed. Results. As a result of the RSL, the appropriate methodology for teaching educational material is the Ignatian Pedagogical Paradigm, using multimedia, images and text, and videos to be viewed on cell phones when used by $100 \%$ of university students, through the social network with the most use in $90 \%$, Facebook. To corroborate the results obtained in the RSL, a survey was applied to $11 \%$ of students enrolled in the ESPOCH in the academic period April - September 2021, which covers 14 questions about the use of social networks. Conclution. This study shows the design process for the development and dissemination of educational material for ESPOCH students based on the agroecological products of the Maquita Foundation, considering the educational methodology of the Ignatian Pedagogical Paradigm (IPP), for the dissemination of educational material in digital channels.

Keywords: educational material, social networks, multimedia, polytechnic students, Ignatian pedagogical paradigm.

\section{Resumen.}

Introducción. En el marco del proyecto multidisciplinario de vinculación "ESPOCH, universidad latinoamericana por el Comercio Justo", la facultad de Informática y Electrónica de la Escuela Superior Politécnica de Chimborazo (ESPOCH), planificó el desarrollo de un material educativo para concientizar a los estudiantes politécnicos acerca del comercio justo y motivarles a que conozcan los productos agroecológicos utilizando canales digitales. Objetivo. Desarrollo de UN MATERIAL EDUCATIVO ACERCA DEL CONSUMO SALUDABLE DE PRODUCTOS DE LA FUNDACIÓN MAQUITA.

Metodología. Se planteó un proceso de diseño del material educativo de tres fases, partiendo en la fase 1 de la selección de una metodología educativa adecuada para lo cual se aplicó Revisión Sistemática de Literatura (RSL), en la fase 2 se determinó el material educativo a desarrollar y en la fase 3 se planteó el plan de difusión del material educativo. Resultados. Como resultados de la RSL, la metodología adecuada para la enseñanza del material educativo es el Paradigma Pedagógico Ignaciano, utilizando multimedia, imágenes y texto, y videos para visualizarse en los teléfonos celulares al ser utilizados en el $100 \%$ de estudiantes universitarios, a través de la red social con más uso en el $90 \%$, Facebook. Para corroborar los resultados obtenidos en la RSL, se realizó una encuesta aplicada al $11 \%$ de estudiantes matriculados en la ESPOCH en el periodo académico abril - septiembre 2021, que abarca 14 preguntas, acerca del uso de redes sociales. Conclusión. El presente estudio muestra el proceso de diseño para el desarrollo y difusión del material educativo para estudiantes de la ESPOCH en base a los productos agroecológicos de Fundación Maquita, considerando la metodología educativa del Paradigma Pedagógico Ignaciano (IPP), para la difusión del material educativo en canales digitales.

Palabras claves: material educativo, redes sociales, multimedia, estudiantes politécnicos, paradigma pedagógico ignaciano. 


\section{Introducción.}

"La vinculación, es la función de puente por excelencia entre la universidad y la sociedad de la cual forma parte y a la que contribuye a dar forma... la vinculación con la sociedad conlleva la misión de integrar las funciones sustantivas de las instituciones de educación superior y de ahí viene su importancia radical... su alcance se extiende al beneficio social directo que la universidad debe reportar a su entorno, ella se debe al entorno y, al mismo tiempo, proviene de él. La relación es de doble vía y la ley no hace más que ratificar el papel preponderante de la vinculación como razón de existir de las instituciones de educación superior." (CACES, 2020)

Es innegable la importancia de la vinculación de la educación superior en el desarrollo de diferentes ámbitos como el del comercio justo y el impulso a la comercialización de productos de fundación Maquita, a la vez que se concientiza a los estudiantes de la importancia de la alimentación saludable y su contribución a la comercialización de productos agroecológicos o ecológicos, como la IFOAM (2008) lo define: "La agricultura ecológica es un sistema de producción que mantiene la salud de los suelos, ecosistemas y personas. Se basa en procesos ecológicos, biodiversidad y ciclos que se adaptan a las condiciones locales, antes que en el uso de insumos con efectos adversos. La agricultura eco- lógica combina tradición, innovación y ciencia para beneficiar el medio ambiente común y promover relaciones justas y una buena calidad de vida para todos los implicados."

En este contexto la ESPOCH, mantiene sendos convenios con organismos seccionales, fundaciones, etc., que le permiten poner su contingente al servicio de la comunidad. El convenio que mantiene la ESPOCH con fundación Maquita desde 2016 impulsa el consumo de productos agroecológicos desde diferentes disciplinas, y es por ello que la facultad de Informática y Electrónica desde el proyecto multidisciplinario de vinculación que inició mediante la aprobación de consejo politécnico, según resolución 122.CP.2020 del 18 de febrero del 2020, "ESPOCH, universidad latinoamericana por el Comercio Justo" desarrolló un MATERIAL EDUCATIVO ACERCA DEL CONSUMO SALUDABLE DE PRODUCTOS DE LA FUNDACIÓN MAQUITA (Maquita, n.d.). Para ello planteó un proceso de diseño del material educativo, de tres fases como se observa en la figura 1, que se aplica en el estudio.

Figura 1. Proceso de Diseño del Material Educativo

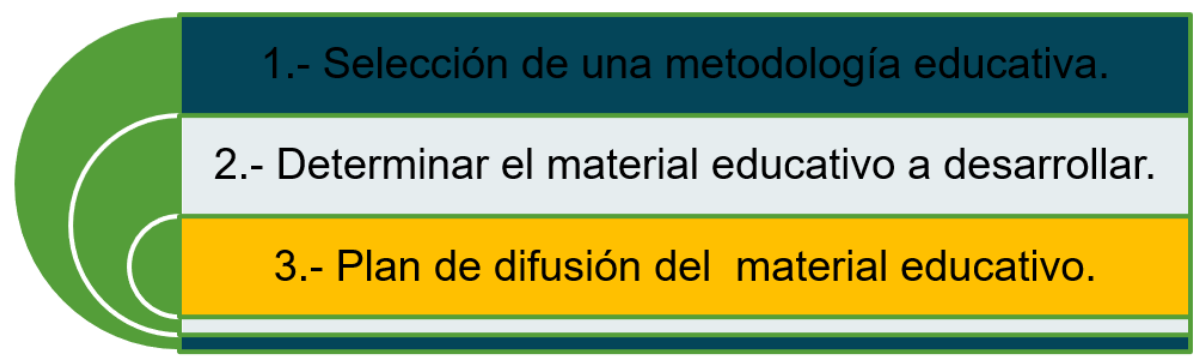

Fuente: Elaboración propia. 


\section{Desarrollo}

\section{Fase 1, Selección de una metodología educativa}

Se realizó una revisión sistemática de literatura ó RSL por sus siglas en inglés, aplicando la metodología de (Kitchenham \& Charters, 2007) partiendo de una búsqueda en la base de datos SCOPUS realizada el 8 de julio del 2020, en base a la cadena de búsqueda en el idioma inglés de: material educativo o metodología de enseñanza, gente joven, presente; obteniéndose como resultado diez artículos que se analizaron para identificar la metodología de enseñanza más adecuada para estudiantes universitarios.

De la RSL, se puede resaltar a (Novita Dewi, 2019) que determina que, en esta era multimedia, los objetivos de la educación se orientan principalmente a aumentar la eficiencia y la productividad al hacer que los jóvenes funcionen en la economía global actual incluyendo humanismo y reflexión en la educación, por ejemplo, en la enseñanza de la literatura.

En éste caso se resalta el estudio descriptivo explicativo que investiga cómo la multimedia puede ayudar a los estudiantes a ser creativos y reflexivos al mismo tiempo enriqueciendo múltiples perspectivas al utilizar "el paradigma pedagógico ignaciano, por sus siglas en inglés IPP, donde se muestra que la multimedia puede ayudar a los estudiantes a pensar más allá de la literatura y ampliar su cognición; el uso de videos, creadores visuales basados en la web y otros recursos multimedia pueden apoyar la creatividad de los estudiantes".(Dewi, 2019)

Además, Sudiarja (2015) considera que la educación debe reformular continuamente su identidad para equilibrar el ritmo acelerado de la cultura global. En ésta era de clonación, Inteligencia Artificial y realidad virtual, la tecnología puede superar a la humanidad (Janicaud 2005), por lo que la educación tiene el deber de trascender su humanidad para hacer que los seres humanos se vuelvan más humanos (Sastrapratedja 2009, Sastrapratedja 2015 ), para ello se sugiere..."un enfoque humanista y contextual, como el paradigma pedagógico ignaciano, es decir, un modelo de aprendizaje-enseñanza con tres componentes clave: Competencia, Conciencia y Compasión (las 3C). Para alcanzar las 3C, los estudiantes siguen los 5 pasos que comprenden Contexto, Experiencia, Reflexión, Acción y Evaluación que conforman un ciclo continuo de aprendizaje”. Como se observa en la figura 2. Paradigma Pedagógico Ignaciano (Borgo, 1990)

Figura. 2 Paradigma Pedagógico Ignaciano

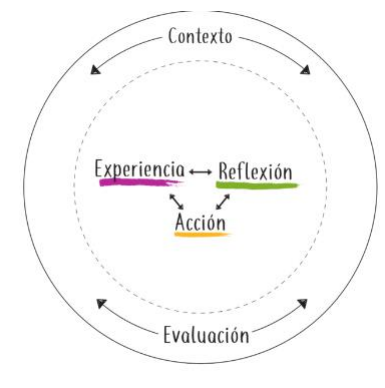

Fuente: (Borgo, 1990) 
Este paradigma de aprendizaje coincide con el estilo de aprendizaje de los Millennials que son expertos en tecnología y fáciles de colaborar, conectarse y crear un cambio social (Pinder-Grover y Groscurth 2009). La enseñanza se complementa con prácticas reflexivas (Dewi2018a) para desarrollar los 3C de los estudiantes de manera completa y creativa. Varios estudios han demostrado que el IPP es aplicable a la enseñanza de diversas materias en disciplinas como la ética empresarial (Van Hise 2013), enfermería (Pennington et al. 2013) y, literatura (Aji 2016).

En base a los resultados de la Revisión Sistemática de Literatura, se propone "Adaptar la metodología de enseñanza IPP para que se genere el proceso de aprendizaje del MATERIAL EDUCATIVO ACERCA DEL CONSUMO SALUDABLE DE PRODUCTOS DE LA FUNDACIÓN MAQUITA".

Para aplicar el IPP, se indagará en el contexto del estudiante de la ESPOCH, el uso de redes sociales y la forma en la que prefiere se le entregue la información, para en base a éstos resultados, desarrollar un material educativo adecuado que pueda ser difundido a los estudiantes de la ESPOCH, mediante una campaña basada en el IPP, que genere en el estudiante conciencia de la importancia del consumo responsable de productos agroecológicos con una nutrición adecuada que le permita también mejorar su salud.

\section{Fase 2, Determinar el material educativo a desarrollar}

De la RSL se obtiene la importancia de la utilización de material multimedia como videos, combinado con texto e imágenes, pues contribuye ampliamente en el aprendizaje, desarrolla creatividad y reflexión (Dewi, 2019). Considerando los productos agroecológicos que la Fundación Maquita comercializa en la provincia de Chimborazo, se determinó el material educativo a desarrollarse es una "Guía de nutrición y alimentación saludable".

La guía de nutrición y alimentación saludable se desarrolló por dos periodos académicos

Durante el periodo académico octubre 2020 - marzo 2021 se trabajó en el desarrollo de este producto, que incluye recetas en base a los productos comercializados por fundación Maquita en la provincia de Chimborazo, con la colaboración de la Srta. Andrea Conde, estudiante de la escuela de Nutrición y Dietética de la ESPOCH y la posterior revisión del contenido por la Dra. Patricia Herrera, docente de Nutrición.

El diseño, diagramación del contenido de la guía y propuesta de presentación de la primera versión se desarrolló con los señores practicantes de la escuela de Diseño Gráfico: Norelia Cevallos y Bryan Meza.

En el periodo académico abril - septiembre 20213 se ha trabajado en el Rediseño, diagramación del contenido, editorial adaptada a las necesidades de redes sociales, dispositivo celular, segunda versión, con las señoritas practicantes de la escuela de Diseño Gráfico: Patricia Robalino y Mariuxi Chávez. 


\section{Fase 3, Promoción del Material Educativo}

"La difusión de la investigación constituye una etapa importante, por lo general final, del trabajo efectuado.” Cegarra Sánchez, J. (2012).

La investigación centrada en proyectos, y la importancia de la difusión digital de los resultados, hace necesario el uso de técnicas de gestión de difusión digital, que según Siso, M. (2019), “...pretende conseguir con una efectiva gestión de proyectos es la optimización de los recursos existentes agregando valor al resultado final y, por tanto, a las organizaciones ejecutoras."

Es importante tener en cuenta la forma cómo se presenta la difusión; para ello, se deberá definir a quién va encaminada, qué debe contener, cuándo debe realizarse, dónde se presenta y cómo hacerlo. "Segmentar a la audiencia resulta de gran utilidad para crear estrategias más específicas que capten la atención del público." Carrasco Ortega, Maité. (2020).

De acuerdo con lo señalado por Soler, A. (2016). "Los adolescentes cuentan con un gran interés por el uso de los recursos virtuales, no sólo en lo relacionado con la comunicación, sino para hacer búsquedas de información y creación de contenidos que los identifique frente al mundo y a la vida."

La búsqueda de estrategias para la difusión entre los estudiantes se convierte en una tarea imperante, de acuerdo con lo señalado por Zuccherino, S. (2016), existe nuevos comportamientos que se basan en nuevos hábitos de vida, nuevas formas de socializar, influenciar y ser influenciados; nuevas modalidades laborales, nuevas profesiones, entre otros. En los tiempos actuales que nos ha tocado reinventarnos y convertir la virtualidad como la vía más efectiva de comunicación las redes sociales se han convertido en uno de los canales estratégicos de difusión.

Sponselee (2016) señala las seis ventajas del uso de las redes sociales en la gestión de proyectos, resumiendo en las siguientes: “...permiten disponer de información en tiempo real sobre el proyecto, dan soporte al desarrollo interactivo e incremental de los proyectos, mejoran de forma eficiente los proyectos, simplifican el trabajo a distancia, facilitan el branding, y estimulan la creación de contenido... Según Torres-Salinas y CabezasClavijo (2008) los servicios, herramientas y plataformas que se crean en el contexto de la web 2.0 facilitan la generación, publicación e intercambio de contenidos en múltiples formatos (videos, fotografías, audio, texto) por parte de los usuarios, sin que estos tengan que poseer competencias digitales avanzadas y, por tanto, están pensadas para su uso por la gran masa de usuarios."

El branding según Paulino Sulz (Suiz, 2020), es el trabajo de gestión de marca con el objetivo de hacerla conocida, deseada y con una imagen positiva en la mente y el corazón de los consumidores. El branding o brand management, implica acciones relacionadas con el propósito, los valores de la marca y el posicionamiento, creando una conexión con el público para influir en sus decisiones de compra. 
Uno de los medios para difundir información son las redes sociales. Según Laudon, K y Guercio, C. (2009) "El comercio electrónico entró en un periodo de reinvención en 2006, con el surgimiento de las redes sociales y las aplicaciones Web 2.0 que atrajeron enormes audiencias en un periodo de tiempo muy corto.". Señala también que: "Los sitios de redes sociales son el equivalente de las redes de palabra fuera de línea, que son la influencia más poderosa sobre el comportamiento de los clientes... la publicidad en las redes sociales es la forma más grande y de más rápido crecimiento de marketing social..."

Con estas premisas el análisis debe enfocarse en los estudiantes de la Escuela Superior Politécnica de Chimborazo, a quienes va dirigida la guía nutricional, y la difusión en el período ordinario de clases, buscando los espacios adecuados donde los estudiantes no tengan sobrecarga de tareas para que se capte su atención de mejor manera.

Para realizar este proceso, es importante conocer al público, sus características y contexto para difundir la información adecuadamente y que se realice el proceso de aprendizaje.

Del análisis en la RSL, de la tecnología y los estudiantes universitarios(González, 2014) se obtiene que el móvil es una realidad en sus vidas, el 100\% de los estudiantes posee un teléfono móvil, además la red social favorita de los estudiantes universitarios es Facebook en el $90 \%$.

\section{Metodología.}

Adaptación de Metodología Paradigma Pedagógico Ignaciano (IPP) para la difusión del material educativo: guía de nutrición y alimentación saludable

Según el análisis detallado en el Proceso de Diseño del Material Educativo, para su aplicación, se consideró: contexto, experiencia, reflexión, acción, evaluación.

1.- El contexto, es el paso inicial, es garantizar que los alumnos se conozcan a sí mismos, a los demás y a los materiales apropiados para aprender.

Población: estudiantes 17-26 años,

Contexto: Entorno de educación superior,

Materiales: Video, multimedia, imágenes, texto.

2.- La experiencia es el segundo elemento. Aquí, los estudiantes participan en actividades cognitivas y afectivas a través de la respuesta a preguntas relacionadas con sus propias experiencias.

"¿Alguna vez conocí a una persona como la de la historia?"; "¿Cómo trataría con una persona así?" y ayudar a los estudiantes a percibir nuevos hechos, perspectivas, conceptos, etc. Videos, e información del origen de los productos agroecológicos, su elaboración, beneficios. De dónde vienen los productos, son emprendimientos, para llegar a los estudiantes y generar empatía con el pequeño productor. 
3.- La tercera y más esencial parte del IPP es la Reflexión. Es el significado de la experiencia, de naturaleza metacognitiva e implica discreción y a actuar en consecuencia.

El material de difusión va orientado a la reflexión, a la vez que sugerimos los beneficios de la alimentación saludable, apoyamos a los pequeños productores al consumir productos distribuidos por fundación Maquita.

Para ello, se desarrolló dos campañas, una de expectativa y otra de difusión de la información. Éstas incluyen videos, imágenes de dónde provienen los productos de emprendedores agropecuarios.

Se propuso el Slogan "Politécnico si te alimentas bien, te sentirás bien"

4.- El cuarto ciclo es el más difícil: la acción. Las manifestaciones de acciones para hacer el bien o mejorar para otros aquí pueden ser de actividad concreta o "elecciones manifestadas externamente", también pueden ser "elecciones interiorizadas" como comprensión, decisión, creencia, compromiso, etc.

Invitar al consumo de los productos Maquita, y a utilizarlos en nuestra alimentación diaria, incluir dónde se puede adquirir los productos.

5.- Finalmente, la evaluación es el último elemento del IPP. Mide no solo si los estudiantes han aprendido las lecciones, sino también si han crecido de manera madura. Busca evaluar la transformación del alumno.

Encuesta, sugerencias, que serán realizadas una vez concluidas las campañas de difusión del material educativo.

El material educativo, se está difundiendo a través de la fan page de la marca del proyecto de vinculación:" ESPOCH, politécnica latinoamericana por el comercio justo" (Facebook, 2021)

\section{Encuesta sobre medios digitales para estudiantes de la ESPOCH}

El presente estudio tiene carácter descriptivo - exploratorio, considerando que el material educativo se difundirá a estudiantes de la ESPOCH, a través de redes sociales, se realizó una encuesta que abarca 14 preguntas entre datos del estudiante y preguntas de interés para la investigación. Se realizó un muestreo aleatorio, que, según lo señalado por (Casas Anguita, Repullo Labrador, \& Donado Campos, 2003) “En este tipo de muestreo, la población se divide en subpoblaciones, denominadas estratos, en función de las variables que pueden tener influencia sobre las características que se quiere medir." en este contexto la población estudiantil en el período académico Abril - Septiembre 2021 es de 21184, aplicando las encuestas que se realizaron en Microsoft Forms, con preguntas de opción múltiple entre los estudiantes. Se obtuvieron 2431 respuestas que equivale al $11 \%$ de la población catalogándola como población finita que de acuerdo con (López-Roldán \& Fachelli, 2017) "si la muestra necesaria es considerable en relación a la población, se considera finita la población y se han de utilizar factores de corrección de población 
finita." El autor también señala que para obtener una muestra de una población finita se establece que por encima del 10\% se suele considerar necesario, y entre un 5\% y un $10 \%$ recomendable. Por lo que estamos en el rango aceptable para la muestra.

\section{Resultados.}

Una vez aplicadas las encuestas, se obtuvieron los siguientes resultados:

Las primeras cuatro preguntas corresponden a datos personales de los estudiantes, demostrando que se ha recolectado información de estudiantes de todas las facultades y de los diferentes semestres de la institución. Estos resultados se muestran en gráficos estadísticos en base a los porcentajes obtenidos, como se muestra a continuación la figura 3. Selección de género, con un 42,68 \% femenino y 57,32 \% masculino.

Figura 3. Seleccione su género

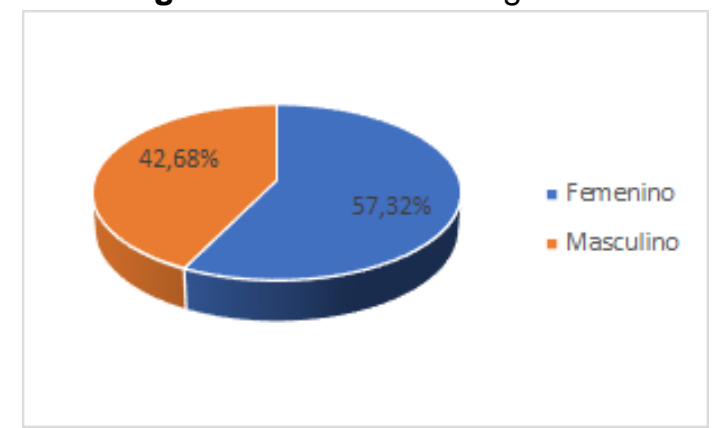

Fuente: Elaboración propia a partir de los datos obtenidos en la encuesta de uso de redes sociales

Figura 4. En qué rango de edad se encuentra, se puede observar los años de los estudiantes que oscilan de los 17 a los 26 en adelante.

Figura 4. En qué rango de edad se encuentra

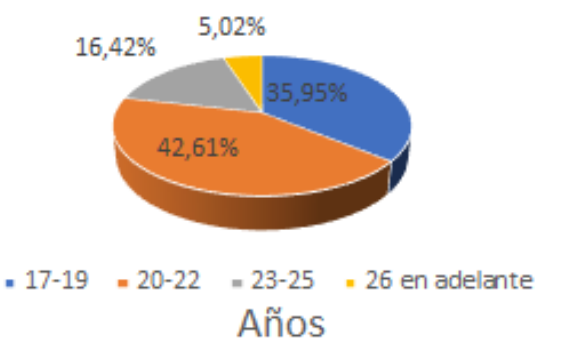

Fuente: Elaboración propia a partir de los datos obtenidos en la encuesta de uso de redes sociales.

La figura 5. Seleccione a que facultad pertenece muestra el porcentaje de pertenencia de los estudiantes. 
Figura 5. Seleccione a que facultad pertenece

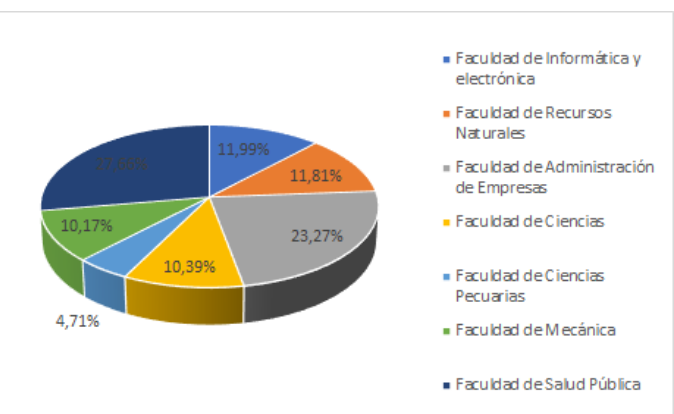

Fuente: Elaboración propia a partir de los datos obtenidos en la encuesta de uso de redes sociales.

La figura 6. Seleccione el semestre en el que se encuentra, se observa los porcentajes desde Nivelación a décimo semestre.

Figura 6. Seleccione el semestre en el que se encuentra

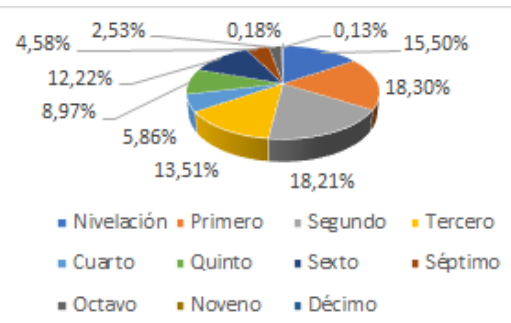

Fuente: Elaboración propia a partir de los datos obtenidos en la encuesta de uso de redes sociales.

Las figuras desde la 7 a la 16, muestran los resultados de preguntas relacionadas al uso del internet y preferencia de redes sociales. De la información obtenida se puede apreciar, en la figura 7 ¿Con qué dispositivos se conecta con mayor frecuencia a internet?, que el $52,12 \%$ de estudiantes utilizan su teléfono celular para conectarse a internet, seguido por computadoras portátiles en un $37,78 \%$, un $8,92 \%$ computadoras de escritorio y un $1.04 \%$ en otros dispositivos.

Figura 7. ¿Con qué dispositivos se conecta con mayor frecuencia a internet?

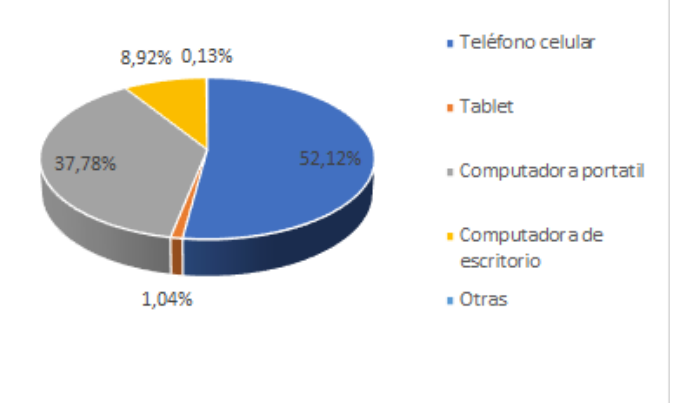

Fuente: Elaboración propia a partir de los datos obtenidos en la encuesta de uso de redes sociales 
En la figura 8. ¿Cuántas horas al día se conecta a internet para revisar sus redes sociales?, demuestra que el $35,52 \%$ de estudiantes se conectan a internet para revisar sus redes sociales de 1 a dos horas al día; el $20.96 \%$ de 2 a 3; el $16.30 \%$ de 4 a 5 horas; el $11.5 \%$ más de 5 horas; el 9,28\% de 3 a 4 horas y el 6,44\% menos de 1 hora al día.

Figura 8. ¿Cuántas horas al día se conecta a internet para revisar sus redes sociales?

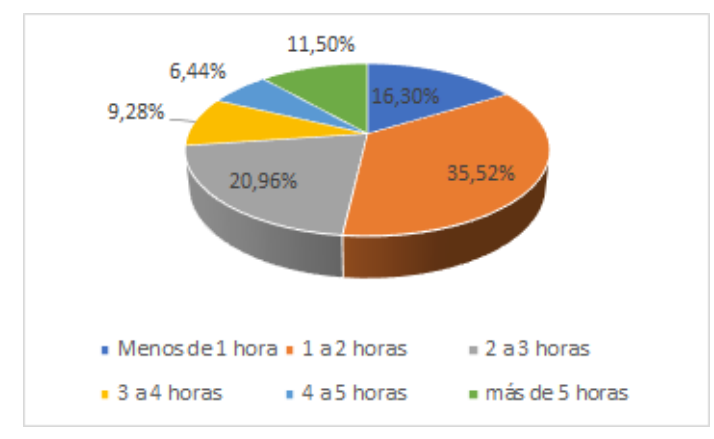

Fuente: Elaboración propia a partir de los datos obtenidos en la encuesta de uso de redes sociales

En la figura 9. En qué horario utiliza con mayor frecuencia el internet para revisar sus redes sociales, se puede apreciar el $54.44 \%$ de estudiantes revisan sus redes sociales en la noche, el $28,67 \%$ en la tarde y el $16,89 \%$ en la mañana.

Figura 9. En qué horario utiliza con mayor frecuencia el internet para revisar sus redes sociales

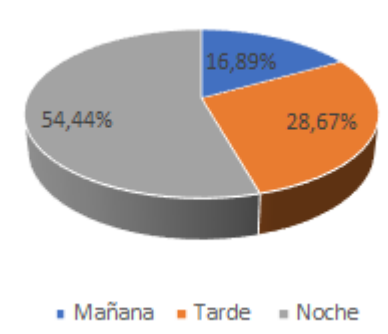

Fuente: Elaboración propia a partir de los datos obtenidos en la encuesta de uso de redes sociales

En la figura 10. Para qué actividad utiliza las redes sociales, los estudiantes hacen uso de las redes sociales en un 36,48\% para informarse; el 23,90\% para revisar noticias; el 23,35 para actividades de ocio; el 9,09\% para compartir contenidos relevantes; el 5,31\% para adquirir productos; y el 1,88\% para otras actividades no especificadas por los entrevistados. 
Figura 10. Para qué actividad utiliza las redes sociales

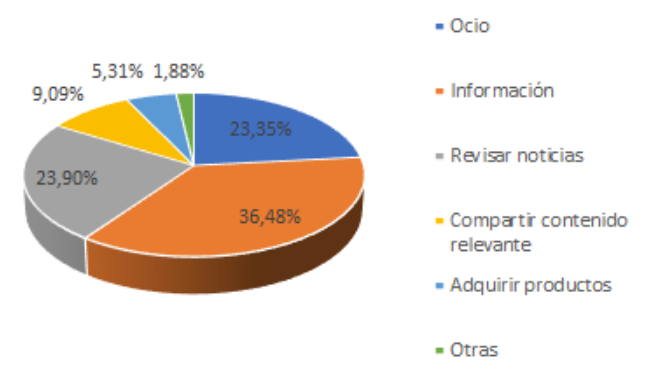

Fuente: Elaboración propia a partir de los datos obtenidos en la encuesta de uso de redes sociales

En la figura 11. Que redes sociales son las utilizadas por usted para informarse, se identificó que la red social más utilizada para informarse es Facebook con un 27,77\%, seguida de WhatsApp con $25,13 \%$, Instagram con un $17,80 \%$, YouTube con un $16,36 \%$, Tik Tok con un $7,45 \%$, Twitter con un $4,47 \%$, Snapchat con un $1,02 \%$.

Figura 11. Que redes sociales son las utilizadas por usted para informarse

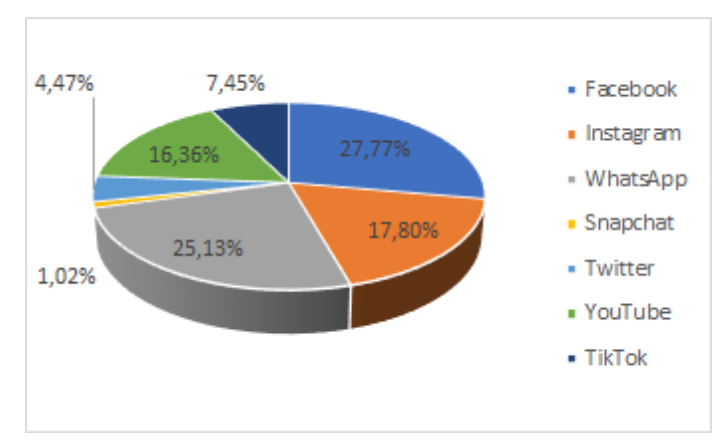

Fuente: Elaboración propia a partir de los datos obtenidos en la encuesta de uso de redes sociales

En la figura 12. Que redes sociales son las utilizadas por usted para adquirir productos, se identificó que la red social más utilizada para adquirir productos es Facebook con un 39.29\%; seguida de WhatsApp con 29.02\%; Instagram con un 25.57\%; YouTube con un $2.70 \%$; Tick Tock con un 2.33\%; Twitter con un $0.78 \%$; Snapchat con un $0.32 \%$.

Figura 12. Que redes sociales son las utilizadas por usted para adquirir productos

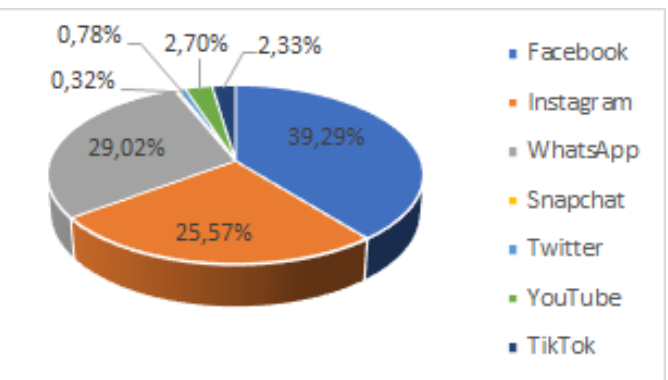

Fuente: Elaboración propia a partir de los datos obtenidos en la encuesta de uso de redes sociales 
En la figura 13. A través de qué tipo de contenido prefiere recibir información, los estudiantes en un $45 \%$ prefieren recibir información a través de imágenes fotografías y texto; un 30,20\% mediante videos; $11,15 \%$ prefiere libros digitales; un 7,65\% Audios; y, un $5,99 \%$ solo texto.

Figura 13. A través de qué tipo de contenido prefiere recibir información

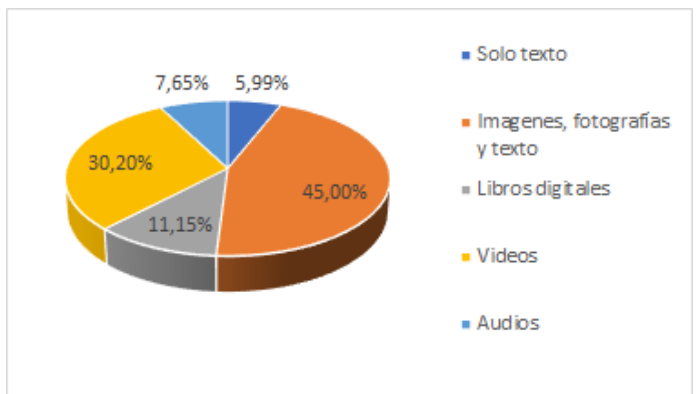

Fuente: Elaboración propia a partir de los datos obtenidos en la encuesta de uso de redes sociales

En la figura 14. De los siguientes temas ¿Cuál consulta con mayor frecuencia en redes sociales?, En referencia los temas que consulta con mayor frecuencia en redes sociales tenemos lo siguiente: Salud 13,14\%; Educación 12,99\%; Diversión 11,90\%; Ciencia 8,56\%; Alimentación saludable 7,91\%; Medio ambiente 7,14\%; Política 6,82\%; Arte 6,82\%; Moda 6,79\%; Cine 6,65\%; Economía 6,19\%; Compras 4,52\%; y, Otras 0,56\%

Figura 14. De los siguientes temas ¿Cuál consulta con mayor frecuencia en redes sociales?

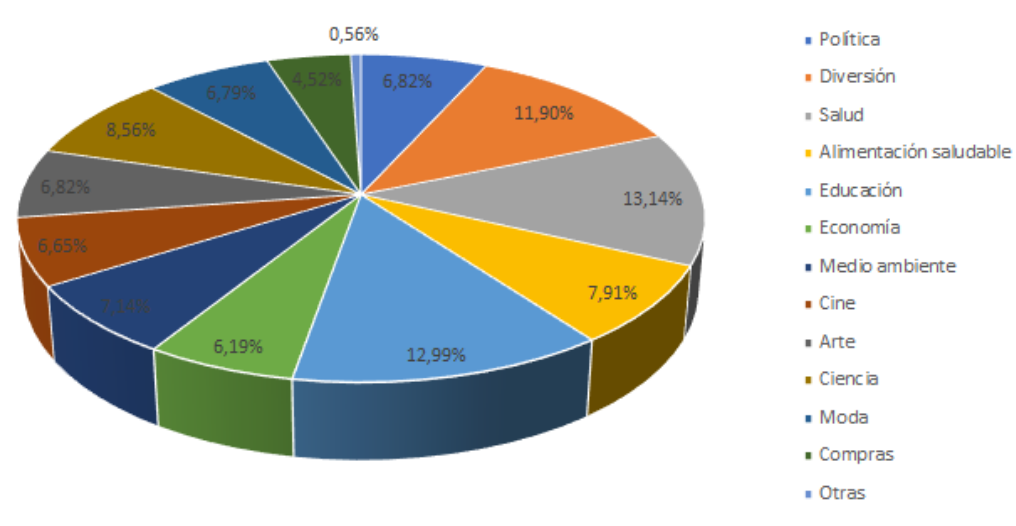

Fuente: Elaboración propia a partir de los datos obtenidos en la encuesta de uso de redes sociales

En la figura 15. A partir del confinamiento generado por la pandemia en el año 2020, indique el porcentaje de incremento que ha tenido en el uso de redes sociales, para recibir información. Se observa que el incremento del $25 \%$ se presenta en el 12,42\% de encuestados, el incremento del $50 \%$ en un $35,13 \%$, el aumento de $75 \%$ del uso en el $38,87 \%$, y en incremento del $100 \%$ en el $13,52 \%$ del total de encuestados. Identificando que el mayor porcentaje con incremento de uso de redes sociales para recibir información es en un $75 \%$. 
Figura 15. A partir del confinamiento generado por la pandemia en el año 2020, indique el porcentaje de incremento que ha tenido en el uso de redes sociales, para recibir información

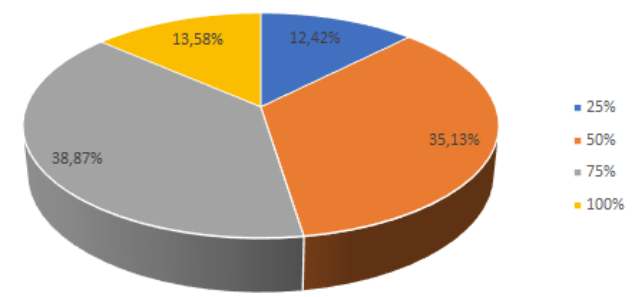

Fuente: Elaboración propia a partir de los datos obtenidos en la encuesta de uso de redes sociales

En la figura 16. A partir del confinamiento generado por la pandemia en el año 2020, indique el porcentaje de incremento que ha tenido en el uso de redes sociales, para adquirir productos online, se ha incrementado en los siguientes porcentajes el 46,14\% de encuestados ha incrementado su uso en un $25 \%$; el $27,24 \%$ de encuestados ha incrementado su uso en un 50\%; el 18,63\% de encuestados ha incrementado su uso en un $75 \%$; y, el 7,99\% de encuestados ha incrementado su uso en un $100 \%$.

Figura 16. A partir del confinamiento generado por la pandemia en el año 2020, indique el porcentaje de incremento que ha tenido en el uso de redes sociales, para adquirir productos online.

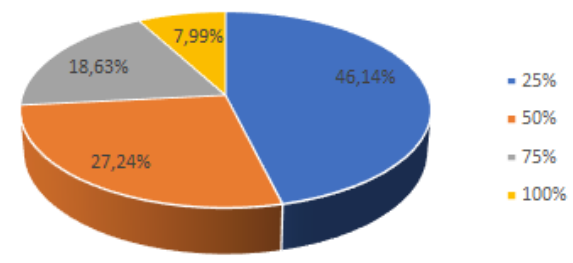

Fuente: Elaboración propia a partir de los datos obtenidos en la encuesta de uso de redes sociales

De los resultados obtenidos se puede evidenciar que el dispositivo electrónico más utilizado por los estudiantes para conectarse a internet es el teléfono celular, por lo tanto, el contenido de la guía nutricional deberá poder visualizarse claramente en la pantalla de este dispositivo, con la finalidad de que puedan recibir la información de manera adecuada.

Adicional a ello, se establece que la mayoría de los estudiantes revisan redes sociales de 1 a 2 horas diarias en el horario de la noche, por lo que deberá considerar difundir el material en este horario. La red social más usada es Facebook, seguida del canal digital WhatsApp. Este resultado concuerda con lo señalado por Soler (2016), actualmente lo 
más utilizado por adolescentes es Facebook y WhatsApp, ya que permitir interactuar de manera más dinámica, asequible y de bajo costo. Estás dos medios digitales son utilizadas con mayor porcentaje para informarse y en un menor nivel para adquirir productos.

Conforme a la información proporcionada por los estudiantes la manera de difundir el material educativo desarrollado es a través de publicaciones en redes sociales que contengan imágenes, fotografías texto, y videos, resultados que concuerda con lo obtenido en la Fase 1. del Proceso de Diseño del Material Educativo.

Los estudiantes revisan diferentes tópicos en redes sociales de los temas analizados tenemos en primer lugar salud, seguido por educación y diversión; y dejando en quinto lugar la alimentación saludable, por lo tanto, se deberá impulsar una campaña de concientización enfocado en los beneficios que brinda la alimentación saludable y cómo estos contribuyen a mejorar la salud y calidad de vida de las personas, a la vez que se difunde el material educativo.

Por último, se debe resaltar el incremento notable que ha tenido el uso de redes sociales para informarse, como cita Soler, A. (2016). "Los adolescentes cuentan con un gran interés por el uso de los recursos virtuales, no sólo en lo relacionado con la comunicación, sino para hacer búsquedas de información y creación de contenidos que los identifique frente al mundo y a la vida." Esto nos ayudará a llegar a los estudiantes politécnicos con el material educativo, generando una amplia socialización, contribuyendo a la mejora en su alimentación y a la comercialización de los productos agroecológicos de fundación Maquita.

Además, es importante observar que, al ser compartido el material educativo y difundido en redes sociales, se podrá llegar a una mayor población, que a la considerada en este trabajo.

\section{Conclusiones.}

- El aplicar Revisión Sistemática de literatura (RSL), metodología propuesta por (Kitchenham \& Charters, 2007) en la búsqueda de la base de datos SCOPUS, para el desarrollo de la Fase 1, selección de una metodología educativa del Proceso de Diseño del Material Educativo, permitió identificar el Paradigma Pedagógico Ignaciano (IPP) y adaptarla en la enseñanza del material educativo "guía nutricional y alimentaria para estudiantes de la ESPOCH".

- La RSL, identificó los elementos más adecuados para generar aprendizaje en los estudiantes, siendo la: multimedia, e información en imágenes, texto, video, los preferidos para el aprendizaje en medios digitales, seleccionándose Facebook, por ser el más utilizado por estudiantes universitarios, junto al celular como dispositivo preferido. Estos resultados fueron corroborados con una encuesta de 14 preguntas, acerca del uso, preferencia, contenido aplicada a 2431 estudiantes 
matriculados en la ESPOCH en el periodo académico abril - septiembre 2021, que corresponde al $11 \%$ de la población general en éste periodo académico.

- En la fase 2 del Proceso de Diseño del Material Educativo se determinó una "Guía de nutrición y alimentación saludable", considerando los productos agroecológicos que la Fundación Maquita comercializa en la provincia de Chimborazo, utilizando textos, imágenes y videos que genere expectativa en el estudiante.

- En la fase 3 del Proceso de Diseño del Material Educativo, se desarrolló el plan de difusión que propone: planificar, determinar formatos, canales de difusión de contenido y medición que permitan mejoras en el contenido difundido, en base a los resultados obtenidos en la RSL y en la encuesta aplicada a los estudiantes de la ESPOCH, aprovechando el incremente en el uso de redes sociales del 75\% a partir del confinamiento generado por la pandemia desde el año 2020, utilizando el canal digital Facebook en la noche, que es el horario preferido para la revisión de redes sociales, con material informativo que se visualice fácilmente en el teléfono celular y computadora portátil, al ser los dispositivos tecnológicos más utilizados por los estudiantes universitarios.

- Los resultados obtenidos en el presente estudio permitieron generar un adecuado proceso para el desarrollo del material educativo que invite a los estudiantes de la ESPOCH a mejorar su alimentación al consumir productos agroecológicos comercializados por fundación Maquita.

\section{Referencias bibliográficas.}

CACES. (2020). Educación Superior y Sociedad. Que pasa con su vinculación?

Casas Anguita, J., Repullo Labrador, J. R., \& Donado Campos, J. (2003). La encuesta como técnica de investigación. Elaboración de cuestionarios y tratamiento estadístico de los datos (I). Atencion Primaria, 31(8), 527-538. https://doi.org/10.1157/13047738

Facebook. (2021). (20+) Espoch - Politécnica Latinoamericana por el Comercio Justo | Facebook. Retrieved August 14, 2021, from https://www.facebook.com/espochcomerciojusto/

González, C. (2014). Estrategias para trabajar la creatividad en la Educación Superior pensamiento de diseño, aprendizaje basado en juegos y en proyectos. RED. Revista de Educacion a Distancia, unknown(40), 7-22.

Kitchenham, B., \& Charters, S. (2007). Guidelines for performing Systematic Literature reviews in Software Engineering Version 2.3. Engineering, 45(4ve), 1051. https://doi.org/10.1145/1134285.1134500

López-Roldán, P., \& Fachelli, S. (2017). El diseño de la muestra. Metodología de La Inestigación Social Cuantitativa, (17), 64. 
Maquita, C. E. S. (n.d.). Fundación Maquita. Retrieved August 5, 2021, from https://maquita.com.ec/

Suiz, P. (2020). Branding: qué es y cómo hacer una eficiente gestión de marca. Retrieved August 6, 2021, from https://rockcontent.com/es/blog/branding/

Zuccherino, S. (2016). Social Media Marketing: la revolución de los negocios y la comunicación digital. Buenos Aires, Temas Grupo Editorial. https://elibro.net/es/ereader/espoch/116713?page $=20$.

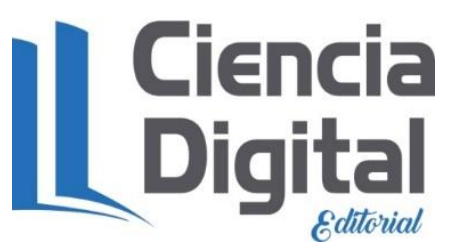




\section{PARA CITAR EL ARTÍCULO INDEXADO.}

Barba Vera, R. G., Maldonado Núñez, A. I., \& Martínez Espinoza, E. F. (2021). Análisis de canales digitales para la distribución de material educativo que promueva el consumo saludable de productos agroecológicos en estudiantes de la ESPOCH . ConcienciaDigital, 4(3.1), 273-290. https://doi.org/10.33262/concienciadigital.v4i3.1.1829

\section{Ciencia \\ LDigital}

El artículo que se publica es de exclusiva responsabilidad de los autores y no necesariamente reflejan el pensamiento de la Revista Conciencia Digital.

El artículo queda en propiedad de la revista y, por tanto, su publicación parcial y/o total en otro medio tiene que ser autorizado por el director de la Revista Conciencia Digital.

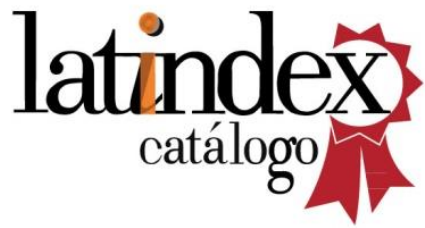

